\title{
Ultraviolet Spectroscopy Used to Fingerprint Five Wild-Grown Edible Mushrooms (Boletaceae) Collected from Yunnan, China
}

\author{
Yan Li, ${ }^{1,2,3}$ Ji Zhang, ${ }^{1,2}$ Tao Li, ${ }^{4}$ Tianwei Yang, ${ }^{5}$ Yuanzhong Wang, ${ }^{1,2}$ and Honggao Liu ${ }^{5}$ \\ ${ }^{1}$ Institute of Medicinal Plants, Yunnan Academy of Agricultural Sciences, Kunming 650200, China \\ ${ }^{2}$ Yunnan Technical Center for Quality of Chinese Materia Medica, Kunming 650200, China \\ ${ }^{3}$ College of Traditional Chinese Medicine, Yunnan University of Traditional Chinese Medicine, Kunming 650500, China \\ ${ }^{4}$ College of Resources and Environment, Yuxi Normal University, Yuxi 653100, China \\ ${ }^{5}$ College of Agronomy and Biotechnology, Yunnan Agricultural University, Kunming 650201, China
}

Correspondence should be addressed to Yuanzhong Wang; boletus@126.com and Honggao Liu; honggaoliu@126.com

Received 22 June 2016; Accepted 24 October 2016

Academic Editor: Khalique Ahmed

Copyright (c) 2016 Yan Li et al. This is an open access article distributed under the Creative Commons Attribution License, which permits unrestricted use, distribution, and reproduction in any medium, provided the original work is properly cited.

\begin{abstract}
Nowadays, wild-grown edible mushrooms which are natural, nutritious, and healthy get more and more popular by large consumers. In this paper, UV spectra of different Boletaceae mushrooms with the aid of partial least squares discriminant analysis (PLS-DA) and hierarchical cluster analysis (HCA) were shown to be a practical and rapid method for discrimination purpose. The specimens of Boletus edulis, Boletus ferrugineus, Boletus tomentipes, Leccinum rugosiceps, and Xerocomus sp. were described based on the UV spectra. From the results, all the specimens were characterized by strong absorption at the wavelengths of 274 and $284 \mathrm{~nm}$ and showed the shoulder at $296 \mathrm{~nm}$. However, changes could be seen in the peak heights at the same wavelength for different samples. After analyzing by chemometrics, visual discrimination among samples was presented and the relationships among them were also obtained. This study showed that UV spectroscopy combined with chemometrics methods could be used successfully as a simple and effective approach for characterization of these five wild-grown edible mushrooms at species and genus levels. Meanwhile, this rapid and simple methodology could also provide reference for the discrimination of edible mushrooms.
\end{abstract}

\section{Introduction}

Wild-grown edible mushrooms are considered as healthy food sources and have long been attracting a great deal of interest by humankind for the natural and nutritional effects [1]. Some species of them are consumed as a delicacy and constitute an increasing share in the world diet [2]. The consumption of wild-grown edible mushrooms is $5.6 \mathrm{~kg}$ of fresh product per household yearly in Czech Republic while higher value is found to be $20-24 \mathrm{~kg}$ in China [3, 4]. Moreover, these mushrooms are not only an important source of revenue for rural economies but also a substantial economic resource in several regions of the world [5].

In the daily life, many edible mushrooms are usually sliced and dried after collecting for better storage and sales.
However, since the market demands for wild-grown edible mushrooms increased, some unscrupulous traders sell inferior mushrooms for profiteering such as mixing different species of dried mushroom slices even toxic ones which lead to unfair competition [6]. In a previous study, Dentinger and Suz [7] analyzed 15 pieces of dried Chinese porcini from a single commercial packet purchased in London and identified three species of mushrooms that have never been formally described by science until now. It suggested that insufficient knowledge of the porcini species contained within food products may pose a health concern. Therefore, for economical, biodiversity-related reasons and so forth, it is often important to discriminate the species of wild-grown edible mushrooms accurately. 
Classical mushroom discrimination is according to careful observation of microscopic and macroscopic morphological characters. However, these methods require trained and experienced people. At present, some analytical techniques for discrimination of edible mushrooms have been published including high-performance liquid chromatography (HPLC), gas chromatography-mass spectrometry (GCMS), infrared (IR) spectroscopy, nuclear magnetic resonance (NMR) spectroscopy, and DNA sequence analyses [8-12]. Since these techniques provide a nonselective signal, the aid of appropriate chemometrics methods is also necessary for interpreting them $[13,14]$. Nevertheless, some imperfections about these methods have been reported. For example, IR needed the experienced technicians and it was hard to model. GC-MS which was expensive could be used to analyze the substance which had the low boiling point and good thermal stability, merely. The ideal chemical technique for the discrimination of mushroom species would provide rapid and accurate analysis. Recently, ultraviolet (UV) spectrometry that reflect the comprehensive fuzz information of samples should be accessible as discrimination tool in diverse research fields, such as the analysis of foods [15], herb medicines [16], automotive window tints [17], for the advantages like rapidity, simplicity, and low cost. Additionally, Li et al. [18] distinguished wild and cultured Macrocybe gigantea with different storage times based on UV spectroscopy combined with multivariate analyses. As reported by Yang et al. [19] UV spectra fingerprints in combination with chemometrics methods could be used to discriminate different parts of edible mushrooms. The rapid and reliable method of UV spectroscopy has shown huge potential for the analysis of edible mushrooms

Mushrooms in the family Boletaceae with 50 genera and 800 species, which are mainly characterized by soft fleshy context, are important groups in the macrofungi of basidiomycete [20, 21]. They are wildly collected and consumed in the main production areas such as eastern Asia, Europe, and North America [22, 23]. In southwestern China, Yunnan Province which is mild and rainy in summer and autumn providing ideal conditions for fungal growth is one of the most important centers for producing, consuming, and trading Boletaceae mushrooms [24]. Boletus edulis which is one of the most famous delicious edible mushrooms in the world is widely liked by people [25]. Other species such as Boletus tomentipes are generally trading on the market during summer and autumn [26]. Apart from flavor and taste, the fruiting bodies of the wild-grown edible mushrooms in this family are considered sources of proteins, amino acids, vitamins, and carbohydrates, as well as minerals and antioxidants $[1,26]$. In addition to edibleness, many medicinal properties in these mushrooms, such as antioxidation, antitumor, and antibiotic effect, have been claimed [27, 28].

In this study, an analytical method for the discrimination of five wild-grown edible mushrooms (Boletaceae) by UV spectroscopy was established and verified. All the spectroscopic data were analyzed by partial least squares discriminant analysis (PLS-DA) and hierarchical cluster analysis (HCA) that aimed to distinguish different specimens and find correlations among these species. The results represented a detailed report on the differentiation of tested Boletaceae species which may provide a utility of methodology for discriminating wild-grown edible mushrooms rapidly and accurately.

\section{Experiment}

2.1. Materials. For the real samples used in this study, the fruiting bodies of wild Boletaceae including five species were collected from Yunnan Province during the collection season (June to September) in 2011. All the samples were authenticated by Dr. Honggao Liu, College of Agronomy and Biotechnology, Yunnan Agricultural University, and preserved in the specimen room of this university. The sample information is listed in Table 1.

2.2. Apparatus. Apparatus were as follows: UV-2550 UVVis Spectrophotometer (Shimadzu, Japan); DFT-100 type grinder (Zhejiang Wenling City Linda Machinery Company, China); SY3200-T type ultrasonic washer (Shanghai Shengyuan Ultrasonic Equipment Company, China); 100mesh stainless steel sieve (Beijing Zhongxi Tai'an Technology Service Company, China); and AR1140 Electronic Analytical Balance (NJ, USA).

2.3. Sample Preparations. All the samples were cleaned up and dried at the temperature of $50^{\circ} \mathrm{C}$ before analysis. Then all of them were ground to fine powder and passed through a 100-mesh stainless steel sieve. The sieved powders were stored in the labeled Ziploc bags at room temperature until further analysis. In each mushroom species, the mixed mushroom samples $(n=7)$ of the same collection site were used to perform the experiment. $0.1 \mathrm{~g}$ of each mixed powdered sample and $10.0 \mathrm{~mL}$ chloroform (analytical grade) were put into a $25 \mathrm{~mL}$ colorimetric tube and extracted by ultrasonication for $30 \mathrm{~min}$. The extracts were filtered and kept as stock solutions for testing.

2.4. Data Acquisition and Chemometrics Methods. Each stock solution was analyzed by UV-2550 UV-Vis spectrophotometer at $0.2 \mathrm{~nm}$ sampling interval and $1.0 \mathrm{~nm}$ slit width. Scans were collected over a range of 190-400 $\mathrm{nm}$ and each sample was measured in triplicate. The UV spectra were treated by the three groups of average and second derivative, in order to eliminate the solvent interference and increase accuracy of spectra. The number of points for derivative was five and the spectral data were mean centered during the analyzed.

The absorption readings obtained over the spectral points of all the samples were converted into a data matrix using Excel 2007 (Microsoft, USA) with the spectral points as variables represented by columns and the corresponding spectral absorption measurements represented by rows. Then the raw spectral data of all the samples were analyzed by partial least squares discriminant analysis (PLS-DA) and hierarchical cluster analysis (HCA) to evaluate the relationships in terms of similarity or dissimilarity among groups of multivariate data. The two statistical analyses were performed by using SIMCA-P ${ }^{+} 11.5$ (Umetrics, Umeå, Sweden) and SPSS 
TABLE 1: Information of all the samples.

\begin{tabular}{|c|c|c|}
\hline Number & Species & Site of collection \\
\hline 1 & Leccinum rugosiceps (Peck) Singer & Wuhua District, Kunming, Yunnan \\
\hline 2 & Leccinum rugosiceps (Peck) Singer & Qujing, Yunnan \\
\hline 3 & Leccinum rugosiceps (Peck) Singer & Pubei, Yimen, Yunnan \\
\hline 4 & Boletus ferrugineus Schaeff. & Wuding, Yunnan \\
\hline 5 & Boletus ferrugineus Schaeff. & Qujing, Yunnan \\
\hline 6 & Boletus ferrugineus Schaeff. & Wuhua District, Kunming, Yunnan \\
\hline 7 & Xerocomus sp. & Nanhua, Chuxiong, Yunnan \\
\hline 8 & Xerocomus sp. & Yao'an, Chuxiong, Yunnan \\
\hline 9 & Xerocomus sp. & Qujing, Yunnan \\
\hline 10 & Boletus tomentipes Earle & Fuliangpeng, Eshan, Yunnan \\
\hline 11 & Boletus tomentipes Earle & Pu’er, Yunnan \\
\hline 12 & Boletus tomentipes Earle & Xiaojie, Eshan, Yunnan \\
\hline 13 & Boletus tomentipes Earle & Qianchang, Yao'an, Yunnan \\
\hline 14 & Boletus tomentipes Earle & Tianshentang, Nanhua, Yunnan \\
\hline 15 & Boletus tomentipes Earle & Tongchang, Yimen, Yunnan \\
\hline 16 & Boletus tomentipes Earle & Shaqiao, Nanhua, Yunnan \\
\hline 17 & Boletus tomentipes Earle & Pu’er, Yunnan \\
\hline 18 & Boletus edulis Bull. & Baofeng, Jinning, Yunnan \\
\hline 19 & Boletus edulis Bull. & Yulu, Nanhua, Yunnan \\
\hline 20 & Boletus edulis Bull. & Pubei, Yimen, Yunnan \\
\hline 21 & Boletus edulis Bull. & Longchuan, Nanhua, Yunnan \\
\hline 22 & Boletus edulis Bull. & Qianchang, Yao'an, Yunnan \\
\hline 23 & Boletus edulis Bull. & Qianchang, Yao'an, Yunnan \\
\hline 24 & Boletus edulis Bull. & Shaqiao, Nanhua, Yunnan \\
\hline
\end{tabular}

20.0 (IBM Corp., Armonk, USA), respectively. Data were visualized by using the two statistical approaches.

\section{Results and Discussion}

3.1. Selection of Extraction Solvent. Every powdered mushroom sample was taken out to form the mixed sample in order to select the extraction solvent. A total of four different extraction solvents (petroleum ether, chloroform, absolute ethanol, and $0.5 \mathrm{~mol} / \mathrm{L} \mathrm{NaOH}$ ) were used and all reagents were of analytical grade. The number of the absorption peaks was used to validate the most appropriate extraction solvent. As shown in the result in Figure 1, the number of the absorption peaks about the chloroform extract is the highest of all the extracts while others have only one or two absorption peaks. It suggested that chloroform extract may include more constituent information about the mushroom samples to reflect their characteristics and chloroform could be the most appropriate extraction solvent.

3.2. UV Spectra of the Wild Edible Boletaceae Mushrooms. The UV spectra of all the specimens are shown in Figure 2. On account of the detection range of the UV-Vis spectrophotometer, the wavelengths of absorption peaks are arranged from 235 to $400 \mathrm{~nm}$ for the sake of avoiding the spectral noise. It shows that all the specimens have higher overlap rate from 235 to $335 \mathrm{~nm}$ than that of other wavelengths. Every sample has some characteristic absorption peaks to reveal its fingerprint feature. In Figure 2, it indicated that some chemical components appeared to be very similar among these five species of Boletaceae mushrooms because all the specimens are characterized by strong absorption at the wavelengths of 274 and $284 \mathrm{~nm}$ and show the shoulder at $296 \mathrm{~nm}$. However, changes can be seen in the peak heights at the same wavelength for different samples and in the ratios between absorbance values at different wavelengths for the same sample. The UV absorption bands of the presented specimens were usually associated with the presence of different chromophores exemplified in conjugated systems as well as other UV-absorbing systems [29]. To a certain degree, when the substance was in high concentration, the corresponding absorbance was high, too [30]. It suggested that the contents of chemical compositions in different species of mushrooms were variable. This result was in agreement with the reports in previous literatures that the accumulation of chemical composition may be affected by the mushroom species $[31,32]$. These differences may be used to discriminate the Boletaceae specimens.

3.3. Partial Least Squares Discriminant Analysis. PLS-DA is a well-established chemometric approach for supervised analyses based on a PLS model in which the dependent variable 


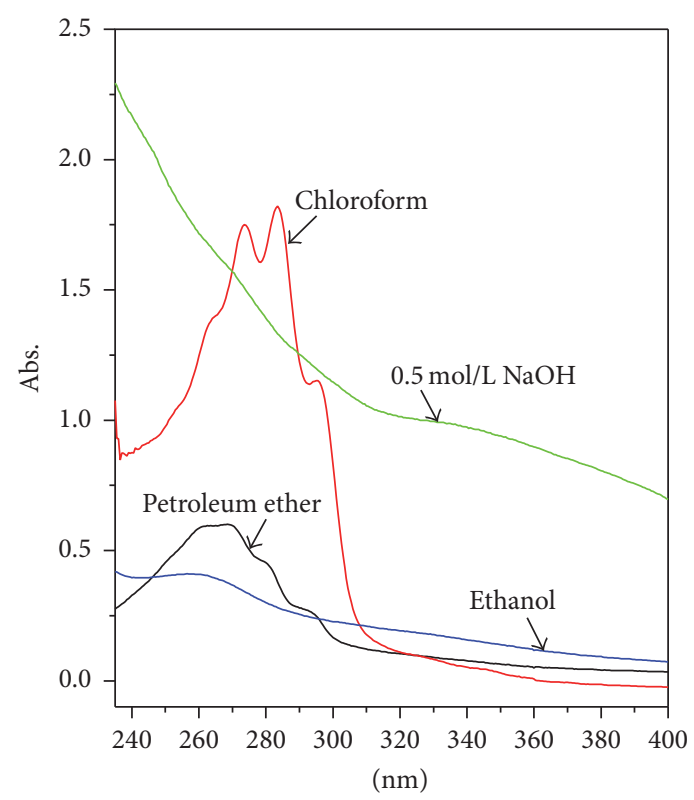

FIGURE 1: UV spectra of different extraction solvents.

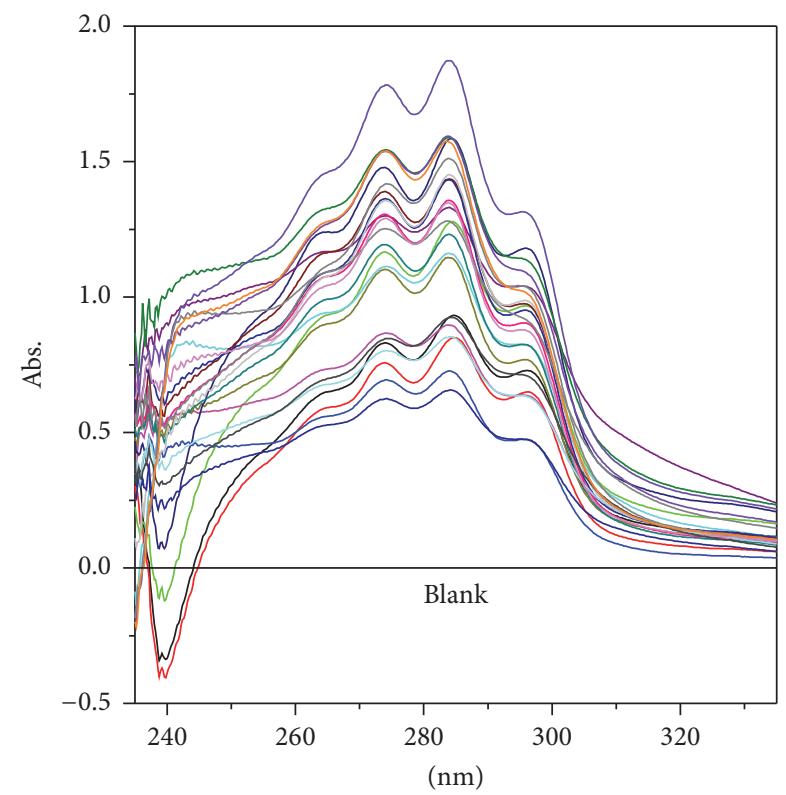

FIGURE 2: UV spectra fingerprints of the wild edible mushrooms (Boletaceae).

(Y block) represents class membership [33]. This method was used as a representative technique to discriminate the mushroom samples according to their species in this study. The first four principal components (PCs) of PLS-DA could explain $85 \%$ of the total variance. The score plot of the sample data with 95\% confidence ellipses is shown in Figure 3. Clear separation of the five species of wild-grown Boletaceae mushrooms is observed in the two-dimensional diagram. The mushroom samples which belong to the same species could form cluster and be distinct with other species. PC 1 is determined mainly by negative scores for the samples of

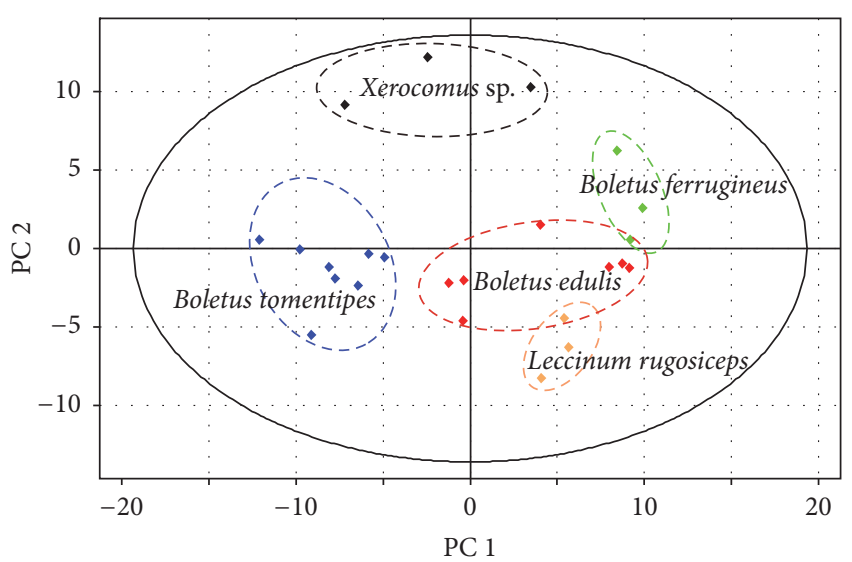

FIgURE 3: PLS-DA score plot based on UV spectra of five species of wild-grown Boletaceae mushrooms.

B. tomentipes while the strongest positive scores on PC 2 are found for samples which belong to Xerocomus sp. All the samples could be distinguished into five classes. Additionally, the individual differences among samples of B. edulis seem to be relatively obvious because these samples are distributed dispersedly. Similarly, clear differences are also visible in the individuals of Xerocomus sp. What is more, the species in the same genus tend to cluster together. As a result, 24 tested samples were classified entirely as their groups by PLS-DA.

According to the spectrographic PLS-DA analysis, a series of scores (variable importance for the projections, or VIPs) were computed to assess the contribution of absorbance to these dimensions. A variable which has the VIP score greater than 1.0 is usually considered important for the discrimination whereas variables with VIPs smaller than 1.0 are less important [34]. Figure 4 shows the contribution of information from each individual variable to the overall samples separation by PLS-DA. As shown in Figure 4, a total of 159 variables have the VIPs greater than 1.0 and about $50 \%$ of them are in the region of $270-300 \mathrm{~nm}$. It showed that the absorption of the wavelength from 270 to $300 \mathrm{~nm}$ was likely to be considered as main factor for discrimination of all the mushrooms. Moreover, in this region, the absorption in the wavelength of $272.4,274.6,278,279.4,280.2,280.4$, 282.2, 283.2, 284, 285, 285.2, 289.8, 291, 292.2, 296, 296.2, $296.4,297.8$, and $298 \mathrm{~nm}$ makes a relatively great contribution because the VIPs of these variables are greater than 2.0 (Table 2).

From the corresponding loading plot (Figure 5), the contribution of different selected variables (VIP $>1.0$ and marked by hollow red square) on the PCs has been recognized. The loadings of variables which made a relatively great contribution (VIPs > 2.0) are shown in Table 2. PC 1 plays a significant role in discriminating $B$. tomentipes and Xerocomus sp. from other samples. Variables in the wavelength of $280.2,289.8$, and $291 \mathrm{~nm}$ have contributions to PC 1, which separate samples number 10 to 17 from the others, with $B$. tomentipes having a negative loading value. The most relevant variables for discriminating Xerocomus sp. from other mushrooms are in the wavelength of $278,279.4,280.4$, 
TABLE 2: VIPs and loadings of selected variables of PLS-DA.

\begin{tabular}{lccc}
\hline Var ID/nm & VIP & Loading 1 & Loading 2 \\
\hline 272.4 & 2.27903 & 0.10182 & -0.034039 \\
274.6 & 2.13471 & 0.0953719 & -0.048547 \\
278 & 2.48458 & -0.111003 & 0.0341494 \\
279.4 & 2.05394 & -0.091763 & 0.0367178 \\
280.2 & 2.2159 & -0.098999 & -0.024235 \\
280.4 & 2.22486 & -0.099399 & 0.0190163 \\
282.2 & 2.24673 & 0.100376 & 0.0255896 \\
283.2 & 2.17515 & 0.0971786 & -0.015926 \\
283.8 & 2.02639 & 0.0905325 & -0.002524 \\
284 & 2.07926 & 0.0928945 & -0.02318 \\
285 & 2.28531 & 0.1021 & -0.017045 \\
285.2 & 2.1474 & 0.0959388 & -0.005978 \\
289.8 & 2.46534 & -0.110143 & -0.039376 \\
291 & 2.15336 & -0.096205 & -0.006975 \\
292.2 & 2.45586 & -0.10972 & 0.0165164 \\
296 & 2.18898 & 0.0977962 & -0.083971 \\
296.2 & 2.08193 & 0.0930139 & -0.03437 \\
296.4 & 2.26702 & 0.101283 & 0.0613701 \\
297.8 & 2.1033 & 0.0939687 & -0.005287 \\
298 & 2.18613 & 0.097669 & 0.0402933 \\
\hline & & &
\end{tabular}

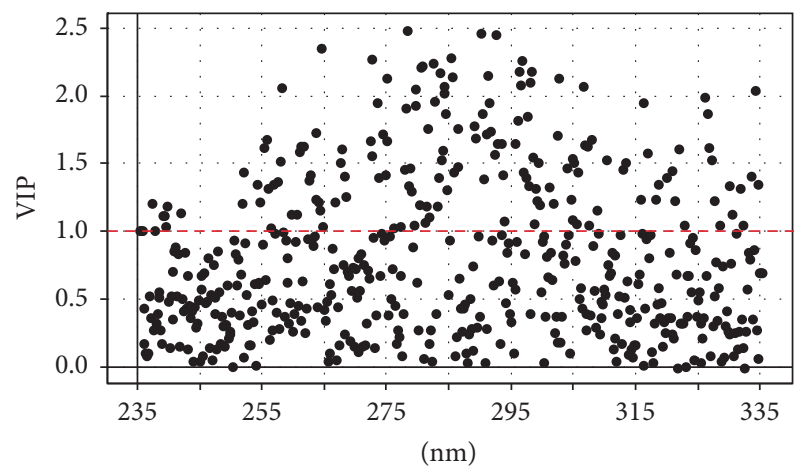

FIgURE 4: Variable Importance for the Projection (VIP) plot of absorbance for the contribution to sample separation from PLS-DA.

and $292.2 \mathrm{~nm}$. In addition, the absorption of wavelength of 282.2, 296.4, and $298 \mathrm{~nm}$ plays the discriminating roles for $B$. ferrugineus from other species while L. rugosiceps and $B$. edulis are in the same quadrant where the absorbance of wavelength of 272.4, 274.6, 283.2, 283.8, 284, 285, 285.2, $296,296.2$, and $297.8 \mathrm{~nm}$ contributes to separating these two species from the other samples.

3.4. Hierarchical Cluster Analysis. HCA is an unsupervised pattern recognition method for clustering samples according to the similarities among them [35]. It was used based on the first four PCs of PLS-DA to classify the samples into groups using the single linkage method for cluster building and the distance between clusters was computed by the squared Euclidean method. As shown in the dendrogram (Figure 6), all the samples have the correct cluster at species level. Overall, all the five species are divided into three main fractions when the distance is 19 and that apparently did reflect interdependent relationships occurring among them. In the first case up to three subfractions can be recognized that relate to $B$. ferrugineus (samples number 4,5 , and 6 ), $B$. tomentipes (samples number 10 to 17), and B. edulis (samples number 18 to 24$)$, respectively, which belonged to genus Boletus, family Boletaceae $[36,37]$. As a consequence, these three species mushrooms had the correct cluster at genus and family levels and corresponded with the fungal classification. Three samples-samples number 7 to 9-are similar and join to form the second cluster. They are all classified as Xerocomus sp. [20]. The last one also contains three mushroom samples. Combined with the information in Table 1, these samples were L. rugosiceps and pertained to genus Leccinum, family Boletaceae [20, 37].

Generally, the different species of edible mushrooms were discriminated based on the morphological characteristics and macroscopic color reactions. However some literatures suggested that these two methods relied on experience and subjective factors $[8,38]$. In addition, the color reactions of the mushrooms may be affected by the environment, climate, agrotype, growing season, and physiological status of the fruiting bodies and this may cause the deviation of subjective judgment [38]. In this study, discrimination of five wild-grown edible mushrooms (Boletaceae) profiled by UV spectroscopy analysis combined with chemometrics allowed for the digitalization of these sample properties providing a novel approach for objective annotation of different edible mushroom (Boletaceae) attributes such as species. In contrast, this analysis was efficient, rapid, and reliable, as based on the chloroform extracts.

PLS-DA was a good tool that could provide an overall look at the initial differences in UV spectra and it was possible to show clear differences in chemical components among the five species of wild-grown edible Boletaceae mushrooms. It suggested that this method could be used to differentiate mushroom specimens according to their species. However, although PLS-DA showed clear separation of the mushrooms, better clustering could be observed in the HCA dendrogram (Figure 6). All the specimens could be distinguished accurately at species level and the relationship among them has been also presented.

Obviously, although there were differences among the five species of wild-grown edible mushrooms, some similar constituents between $B$. edulis and $B$. tomentipes could be reflected on account of these two species samples joined together at first. More interestingly, according to the results of dendrogram, the interspecific differences between $B$. edulis and $B$. tomentipes could be the smallest in B. edulis, $B$. ferrugineus, and $B$. tomentipes even though these three species belonged to the same genus. This may be related to the genetic stability and variability of different species of Boletaceae mushrooms during the long-term evolution process. With regard to the level of genus, Boletus may have similar relationship with Xerocomus based on the chemical analysis 


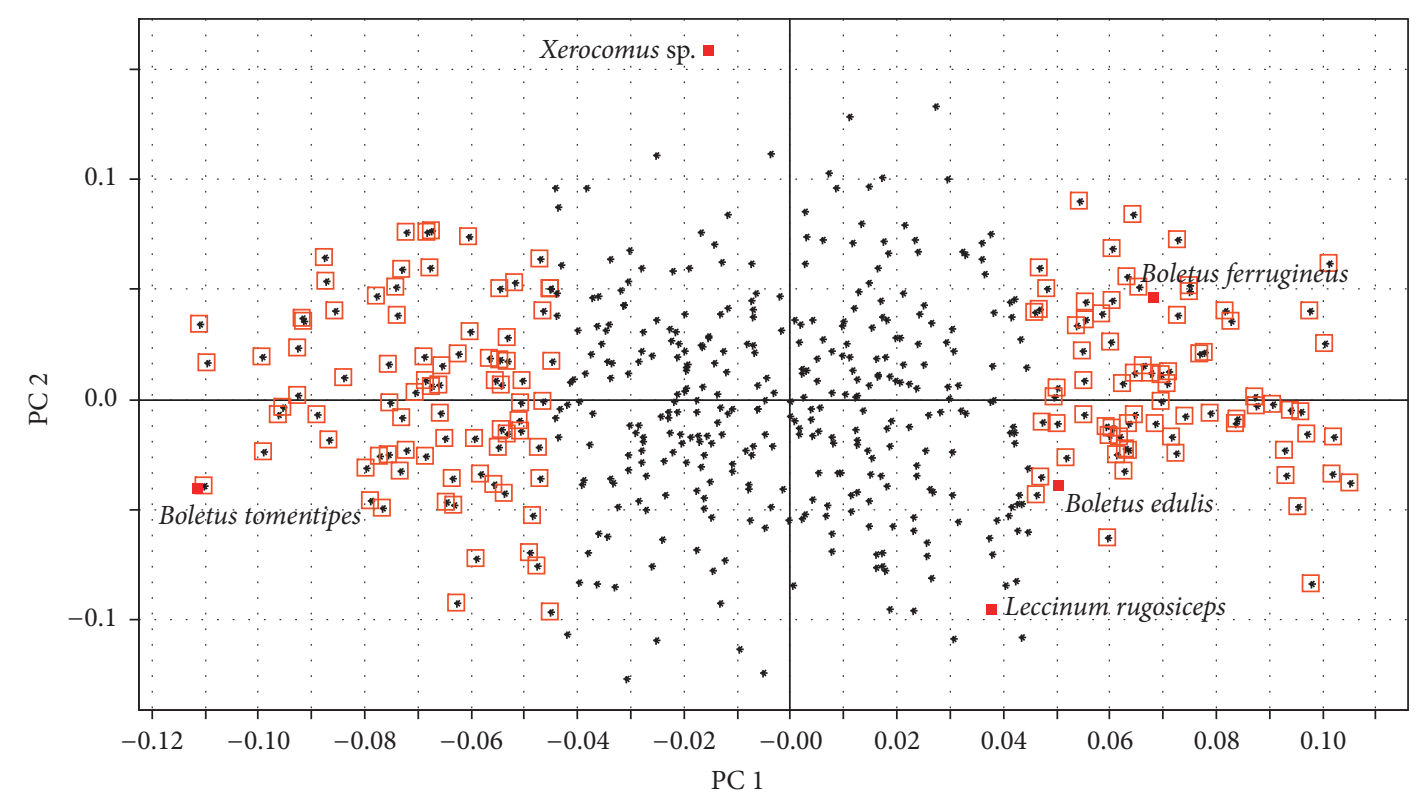

FIgURE 5: Loading plot generated from the PLS-DA model of the mushroom samples.

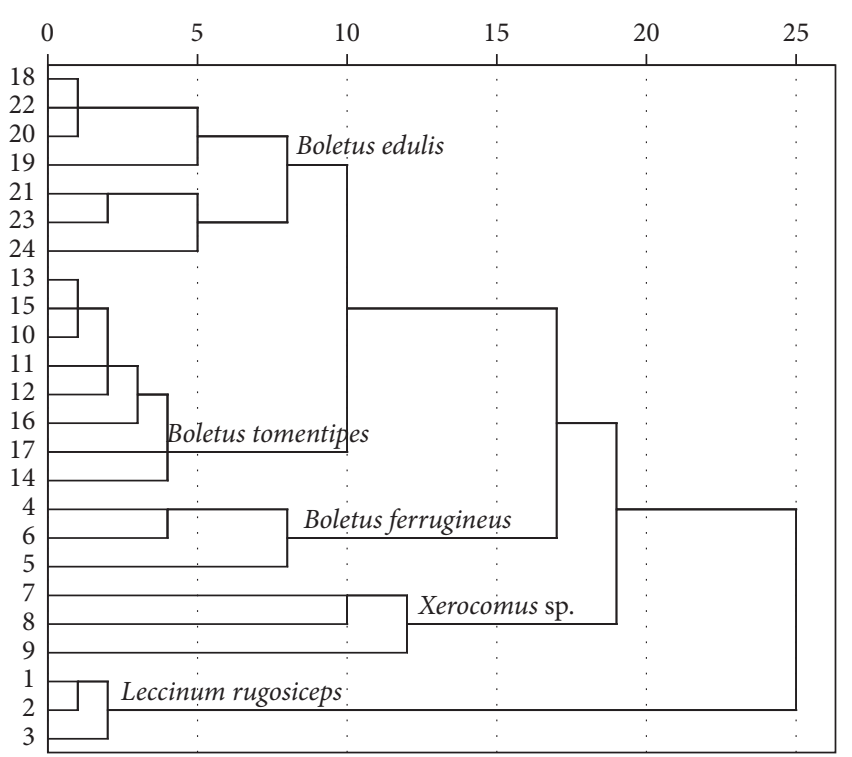

FIGURE 6: Dendrogram resulting from hierarchical cluster analysis.

because these two genera could cluster together firstly among the three genera studied in this study. This was consistent with the previous study that some mycologists have incorporated Xerocomus into Boletus [39]. Additionally, Boletus was clearly different from Leccinum that the samples which belonged to the same genus could be clustered to differentiate with the other one. Indeed, a recent paper has demonstrated this result by molecular analyses that explained this phenomenon in a genetic way [40]. On the other hand, this method for chemical analysis verified the consequence of previous study. It could provide a new way to classify the edible mushrooms.

\section{Conclusions}

This paper described the combination of UV spectroscopy and chemometrics as a rapid discrimination approach of wild-grown Boletaceae mushrooms. The results demonstrated that obvious differences could be found in the whole chemical components based on the chloroform extracts of $B$. edulis, B. ferrugineus, B. tomentipes, L. rugosiceps, and Xerocomus sp. All the samples could be distinguished accurately at species and genus levels and the relationships among them have been presented. On the other hand, this study could offer an economical, effective, and useful discrimination approach of wild-grown edible mushrooms.

\section{Competing Interests}

The authors declare that there is no conflict of interests regarding the publication of this paper.

\section{Acknowledgments}

This work was sponsored by the National Natural Science Foundation of China (31660591, 21667031) and the Science Foundation of the Yunnan Province Department of Education (2016ZZX106).

\section{References}

[1] X.-M. Wang, J. Zhang, L.-H. Wu et al., "A mini-review of chemical composition and nutritional value of edible wildgrown mushroom from China," Food Chemistry, vol. 151, pp. 279-285, 2014.

[2] P. Kalač, "Chemical composition and nutritional value of European species of wild growing mushrooms: a review," Food Chemistry, vol. 113, no. 1, pp. 9-16, 2009. 
[3] L. Šišák, "The importance of mushroom picking as compared to forest berries in the Czech Republic," Mykologický Sborník, vol. 84, no. 3, pp. 78-83, 2007.

[4] D. Zhang, T. Y. Gao, P. Ma, Y. Luo, and P. C. Su, "Bioaccumulation of heavy metal in wild growing mushrooms from Liangshan Yi nationality autonomous prefecture, China," Wuhan University Journal of Natural Sciences, vol. 13, no. 3, pp. 267-272, 2008.

[5] M. De Román and E. Boa, "Collection, marketing and cultivation of edible fungi in Spain," Micologia Aplicada International, vol. 16, no. 2, pp. 25-33, 2004.

[6] Y. M. Shi, G. Liu, Y. L. Sun, S. X. Wei, C. Q. Yan, and X. J. He, "Identifition of Tricholoma matsutake (S. Ito et Imai) Sing and Agaricus Blazei Murrill using Fourier transform infrared spectroscopy and hierarchical cluster analysis," The Journal of Light Scattering, vol. 22, pp. 171-174, 2010.

[7] B. T. M. Dentinger and L. M. Suz, "What's for dinner? Undescribed species of porcini in a commercial packet," PeerJ, vol. 2, article e570, 2014.

[8] G. Liu, J.-H. Liu, A.-M. Yang, Q. Dong, and D.-S. Song, "Identification of edible mushrooms by Fourier transform infrared spectroscopy," Spectroscopy and Spectral Analysis, vol. 24, no. 8, pp. 941-945, 2004.

[9] J. Zhang, X. Zhong, S. Li, G. Zhang, and X. Liu, "Metabolic characterization of natural and cultured Ophicordyceps sinensis from different origins by ${ }^{1} \mathrm{H}$ NMR spectroscopy," Journal of Pharmaceutical and Biomedical Analysis, vol. 115, pp. 395-401, 2015.

[10] L. Barros, M. Dueñas, I. C. F. R. Ferreira, P. Baptista, and C. Santos-Buelga, "Phenolic acids determination by HPLCDAD-ESI/MS in sixteen different Portuguese wild mushrooms species," Food and Chemical Toxicology, vol. 47, no. 6, pp. 10761079, 2009.

[11] R. Malheiro, P. Guedes de Pinho, S. Soares, A. César da Silva Ferreira, and P. Baptista, "Volatile biomarkers for wild mushrooms species discrimination," Food Research International, vol. 54, no. 1, pp. 186-194, 2013.

[12] G. Wu, B. Feng, J. P. Xu et al., "Molecular phylogenetic analyses redefine seven major clades and reveal 22 new generic clades in the fungal family Boletaceae," Fungal Diversity, vol. 69, no. 1, pp. 93-115, 2014.

[13] M. Y. Shen, M. Y. Xie, S. P. Nie, Y. Q. Wan, and J. H. Xie, "Discrimination of different Ganoderma species and their region based on GC-MS profiles of sterols and pattern recognition techniques," Analytical Letters, vol. 44, no. 5, pp. 863-873, 2011.

[14] I. Marekov, S. Momchilova, B. Grung, and B. NikolovaDamyanova, "Fatty acid composition of wild mushroom species of order Agaricales-Examination by gas chromatography-mass spectrometry and chemometrics," Journal of Chromatography B, vol. 910, pp. 54-60, 2012.

[15] P. H. G. D. Diniz, M. F. Barbosa, K. D. T. de Melo Milanez, M. F. Pistonesi, and M. C. U. de Araújo, "Using UV-Vis spectroscopy for simultaneous geographical and varietal classification of tea infusions simulating a home-made tea cup," Food Chemistry, vol. 192, pp. 374-379, 2016.

[16] H. A. Gad, S. H. El-Ahmady, M. I. Abou-Shoer, and M. M. AlAzizi, "A modern approach to the authentication and quality assessment of thyme using UV spectroscopy and chemometric analysis," Phytochemical Analysis, vol. 24, no. 6, pp. 520-526, 2013.
[17] K. J. van der Pal, M. Maric, W. Van Bronswijk, and S. W. Lewis, "Ultraviolet-visible spectroscopic characterisation of automotive window tints for forensic purposes," Analytical Methods, vol. 7, no. 13, pp. 5391-5395, 2015.

[18] Y. Li, J. Zhang, H. Liu, H. Jin, Y. Wang, and T. Li, "Discrimination of storage periods for Macrocybe gigantea (Massee) using UV spectral fingerprints," Czech Journal of Food Sciences, vol. 33, no. 5, pp. 441-448, 2016.

[19] T. W. Yang, B. K. Cui, J. Zhang et al., "Identification of different parts of edible bolete mushrooms by UV fingerprint," Mycosyst, vol. 33, no. 2, pp. 262-272, 2014.

[20] X. L. Mao, The Microfungi in China, Henan Science and Technology Press, Henan, China, 1st edition, 2000.

[21] G.-L. Wang, S.-Q. Wu, and Q.-F. Wu, "Separation, purification and identification of acidic polysaccharide fraction extracted from Boletus edulis and its influence on mouse lymphocyte proliferation in vitro," Journal of Chemical and Pharmaceutical Research, vol. 5, no. 12, pp. 431-437, 2013.

[22] N. Sitta and M. Floriani, "Nationalization and globalization trends in the wild mushroom commerce of Italy with emphasis on porcini (Boletus edulis and allied species)," Economic Botany, vol. 62, no. 3, pp. 307-322, 2008.

[23] L. Sun, M. Yang, X. Bai, and Y. Zhuang, "Effects of different cooking methods on nutritional characteristics of Boletus aereus," Advanced Materials Research, vol. 634, no. 1, pp. 14741480, 2013.

[24] F. K. Zhu, L. Qu, W. X. Fan, M. Y. Qiao, H. L. Hao, and X. J. Wang, "Assessment of heavy metals in some wild edible mushrooms collected from Yunnan Province, China," Environmental Monitoring and Assessment, vol. 179, no. 1-4, pp. 191-199, 2011.

[25] M. Bovi, L. Cenci, M. Perduca et al., "BEL $\beta$-trefoil: a novel lectin with antineoplastic properties in king bolete (Boletus edulis) mushrooms," Glycobiology, vol. 23, no. 5, pp. 578-592, 2013.

[26] T. Li, Y. Wang, J. Zhang, Y. Zhao, and H. Liu, “Trace element content of Boletus tomentipes mushroom collected from Yunnan, China," Food Chemistry, vol. 127, no. 4, pp. 1828-1830, 2011.

[27] R. Gu, "Analysis about the condition of extraction and purification of polysaccharide in Boletus edulis," Farm Machinery, no. 20, pp. 150-151, 2011.

[28] W. Radzki, A. Sławińska, E. Jabłońska-Ryś, and W. Gustaw, "Antioxidant capacity and polyphenolic content of dried wild edible mushrooms from Poland," International Journal of Medicinal Mushrooms, vol. 16, no. 1, pp. 65-75, 2014.

[29] Y. E. Zeng and L. Zhang, Instrumental Analysis, Science Press, Beijing, China, 5th edition, 2010.

[30] K.-L. Wei, Z.-Y. Wen, X. Wu, Z.-W. Zhang, and T.-L. Zeng, "Research advances in water quality monitoring technology based on UV-Vis spectrum analysis," Spectroscopy and Spectral Analysis, vol. 31, no. 4, pp. 1074-1077, 2011.

[31] I. Akata, B. Ergönül, and F. Kalyoncu, "Chemical compositions and antioxidant activities of 16 wild edible mushroom species grown in Anatolia," International Journal of Pharmacology, vol. 8, no. 2, pp. 134-138, 2012.

[32] Z. Zhang, Z. Gu, Y. Yang, S. Zhou, Y. F. Liu, and J. Q. Tang, "Evaluation of the umami taste of three species of dried wild edible fungi," Food Scence, vol. 34, no. 21, pp. 51-54, 2013.

[33] S. Wold, M. Sjöström, and L. Eriksson, "PLS-regression: a basic tool of chemometrics," Chemometrics and Intelligent Laboratory Systems, vol. 58, no. 2, pp. 109-130, 2001. 
[34] N. Shetty, M. H. Olesen, R. Gislum, L. C. Deleuran, and B. Boelt, "Use of partial least squares discriminant analysis on visible-near infrared multispectral image data to examine germination ability and germ length in spinach seeds," Journal of Chemometrics, vol. 26, no. 8-9, pp. 462-466, 2012.

[35] C. Sârbu, R. D. Naşcu-Briciu, A. Kot-Wasik, S. Gorinstein, A. Wasik, and J. Namieśnik, "Classification and fingerprinting of kiwi and pomelo fruits by multivariate analysis of chromatographic and spectroscopic data," Food Chemistry, vol. 130, no. 4, pp. 994-1002, 2012.

[36] X. L. Wu, X. L. Mao, G. E. Tuli et al., Medicinal Fungi of China, Science Press, Beijing, China, 1st edition, 2013.

[37] Index Fungorum, http://www.indexfungorum.org/Names/Names .asp.

[38] H. L. Wei, H. B. Li, L. L. Wang et al., "Molecular recognition of species in Boletus sect. Appendiculati," Mycosyst, vol. 33, no. 2, pp. 242-253, 2014.

[39] P. M. Kirk, P. F. Cannon, D. W. Minter, and J. A. Stalpers, Ainsworth \& Bisby's Dictionary of the Fungi, CAB International, Wallingford, UK, 10th edition, 2008.

[40] T. Riviere, A. G. Diedhiou, M. Diabate et al., "Genetic diversity of ectomycorrhizal Basidiomycetes from African and Indian tropical rain forests," Mycorrhiza, vol. 17, no. 5, pp. 415-428, 2007. 

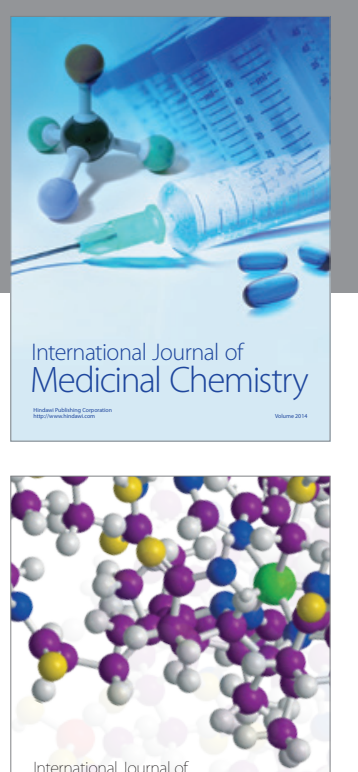

Carbohydrate Chemistry

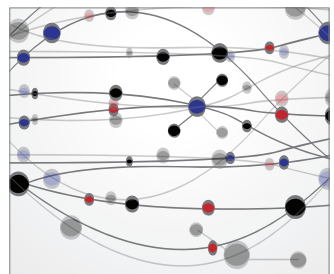

The Scientific World Journal
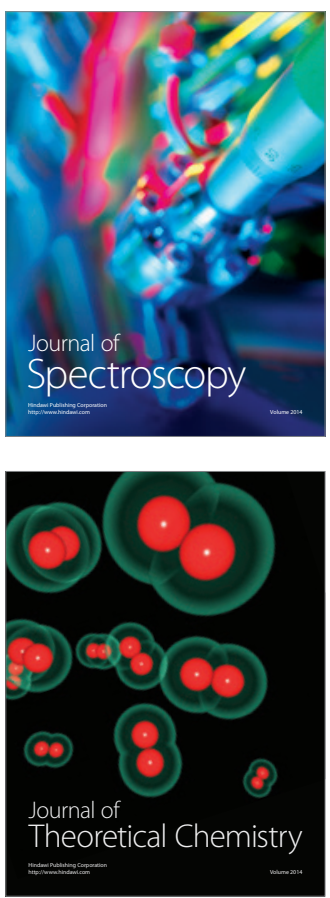
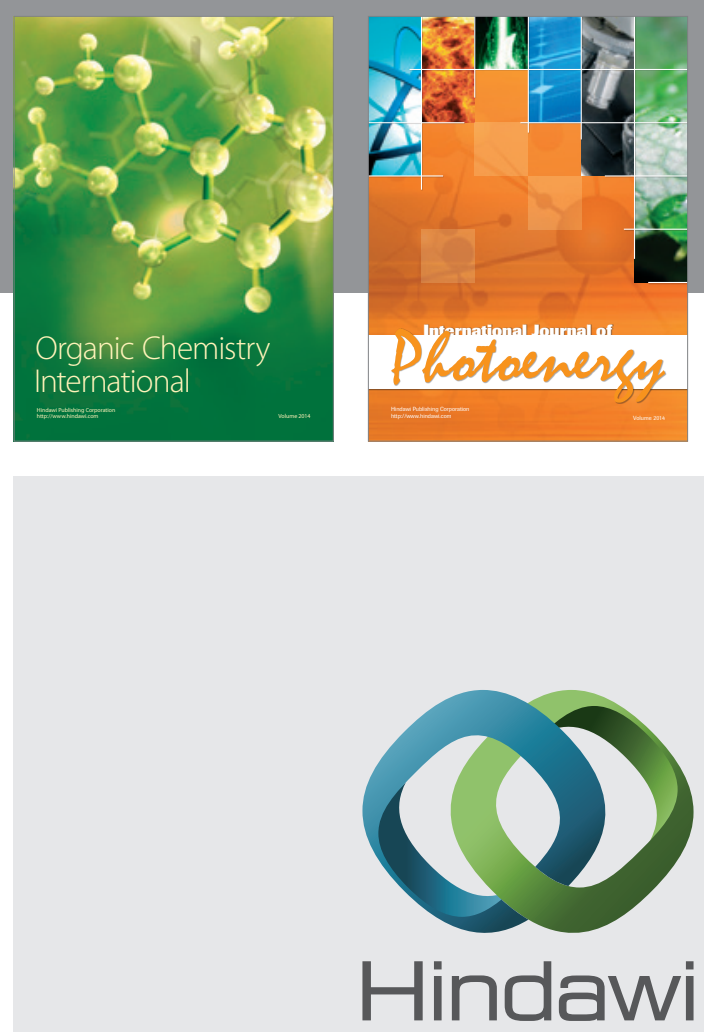

Submit your manuscripts at

http://www.hindawi.com

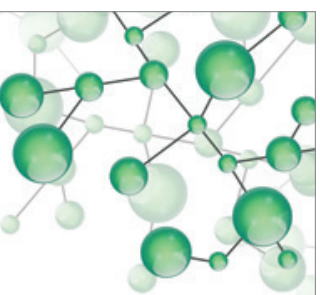

International Journal of

Inorganic Chemistry

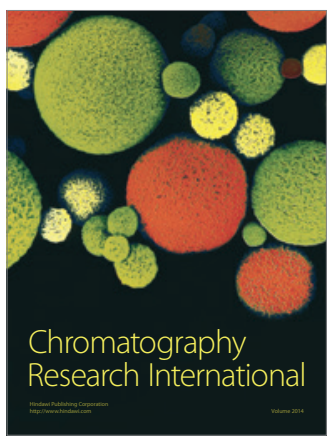

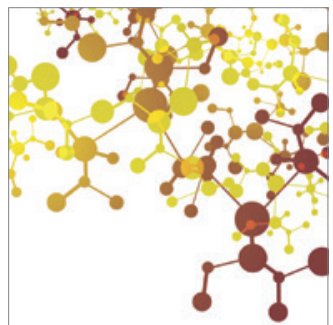

Applied Chemistry
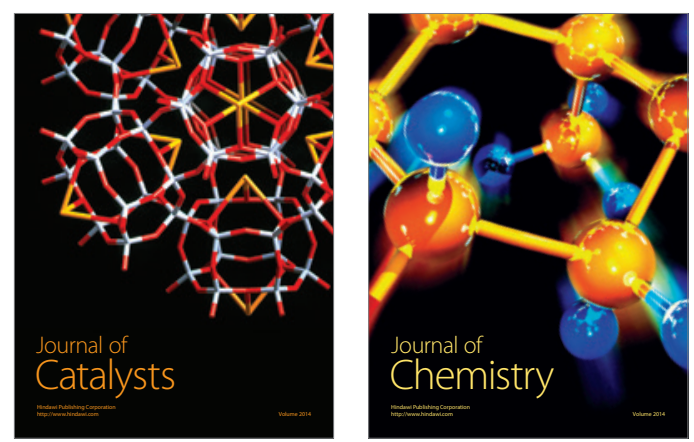
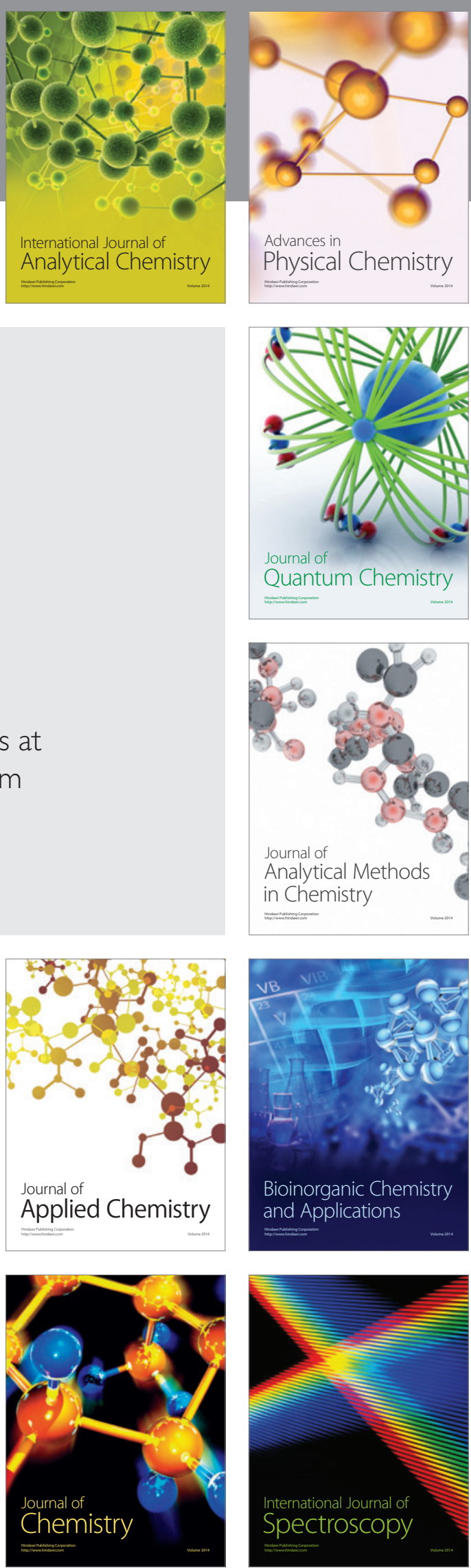